\title{
La fondamentalisation de la protection de l'environnement : conversations entre sociétés chrétiennes mystiques et sociétés autochtones à propos de la nature
}

\section{Pierre-Yves Chicot}

\section{(2) OpenEdition Journals}

Édition électronique

URL : http://journals.openedition.org/add/357

DOI : $10.4000 /$ add. 357

ISSN : 2606-1988

Éditeur

Presses universitaires de Rouen et du Havre

Édition imprimée

Date de publication : 1 juillet 2015

Pagination : 89-110

ISBN : 979-10-240-0516-4

ISSN : 1955-0855

Référence électronique

Pierre-Yves Chicot, «La fondamentalisation de la protection de l'environnement : conversations entre sociétés chrétiennes mystiques et sociétés autochtones à propos de la nature », Les Annales de droit [En ligne], 9 | 2015, mis en ligne le 08 janvier 2018, consulté le 04 mai 2019. URL : http:// journals.openedition.org/add/357 ; DOI : 10.4000/add.357

Ce document a été généré automatiquement le 4 mai 2019.

Presses universitaires de Rouen et du Havre 


\title{
La fondamentalisation de la protection de l'environnement : conversations entre sociétés chrétiennes mystiques et sociétés autochtones à propos de la nature
}

\author{
Pierre-Yves Chicot
}

1 L'adage ubi societas ubi jus témoigne de la prégnance de la règle juridique dans la recherche de la régulation sociale. D'un point de vue fondamental, le droit de l'environnement procède de la protection et de la préservation de la nature. Il signifie, au fond, le respect qui doit lui être voué car elle a précédé l'homme. Le droit de l'environnement introduit mutatis mutandis un rapport hiérarchique entre l'homme et la nature. Dans cette relation homme/nature marquée par une asymétrie, l'avantage de la prééminence revient à la nature du point de vue des "sociétés traditionnelles " qui érigent en «dieu» la Terre, l'univers et les principes créateurs ${ }^{1}$. Cette conception est aussi partagée par les sociétés chrétiennes mystiques.

2 Nous entendons par « sociétés traditionnelles » celles qui ne sont pas ou peu affectées par le processus d'industrialisation de l'économie. L'exemple vraisemblablement le plus illustratif est constitué par les organisations tribales autochtones. La présente étude s'appuiera sur le cas des autochtones d'Amérique. Le succès de la logique industrielle de même que sa diffusion généralisée s'inscrit à bien des égards aux antipodes du respect de la nature.

3 Ainsi émerge, par et dans les sociétés industrielles, la notion de développement durable qui exige de concilier la préservation de la nature tout en garantissant la croissance économique. Le concept de croissance est plutôt étranger aux sociétés traditionnelles qui lui préfèrent l'idée de garantie de subsistance des individus. Le droit de l'environnement qui peut être désigné comme le droit du respect et de la protection de la nature nourricière, puise ses racines dans le droit naturel ${ }^{2}$. 
Un droit naturel ou divin qui s'éloigne au fur et à mesure que la société est gagnée par le progrès technique. Son retour est tout aussi manifeste lorsque l'homme s'incline devant la nature. Il formalise alors des règles de droit protectrices de cette dernière, avec pour objectif final la prospérité de la postérité humaine. Pareille option favorise la promotion de la notion de " générations futures » qui légitime à bien des égards le développement de droits protecteurs de l'environnement (droit de l'eau, droit de l'air, droit relatif à la biodiversité, etc.)

5 Les sociétés traditionnelles, et notoirement les peuples autochtones, sont particulièrement bien indiquées pour ce qui concerne l'accès à des connaissances inhérentes à la nature, dont la maîtrise est ancestrale. La prise en compte de la vision des sociétés traditionnelles dans le processus de fondamentalisation du droit de l'environnement exprime vraisemblablement une rupture.

La conceptualisation rejoint la fondamentalisation du droit de l'environnement, entendue comme un processus qui tend à constituer les bases, l'assise de la matière. Les concepts constituent ainsi les composantes d'une matière juridique évoluée devenue système. Elles sont aussi porteuses de valeurs et révèlent ce qui fait l'essence même de ce droit. L'avènement d'un cadre conceptuel entraîne de véritables mutations, à l'instar de l'émergence du développement durable comme nouveau paradigme du droit de l'environnement. On en arrive alors à une conception davantage humaniste du droit de l'environnement.

7 Cette conception humaniste du droit de l'environnement structure un ordre juridique venant redoubler ou garantir un ordre social désireux de limiter l'encadrement du développement des sciences et des techniques, afin de permettre à celui-ci de prévenir les abus possibles et de limiter les risques.

8 L'irruption de la pensée des sociétés traditionnelles dans la fondamentalisation du droit de l'environnement entraîne un bouleversement des valeurs, osons dire une restauration des valeurs ${ }^{3}$. Sur le plan du droit, on assiste à une révolution juridique dans la mesure où l'inspiration de la sécrétion de la règle ainsi que son contenu ne proviennent plus exclusivement $\mathrm{du}$ pouvoir central étatique, possiblement inspiré par les lobbys industriels. Le changement de paradigme est matérialisé par une autre manière de concevoir le droit de l'environnement associant dès lors de nouveaux acteurs longtemps ignorés.

9 Les conversations entre les sociétés mystiques et les sociétés autochtones qui relèvent davantage de la concorde, mettent en lumière l'homme qui s'incline devant la nature. Pareille attitude favorise l'éclosion d'un droit fondamentalement respectueux de l'environnement (1), mais aussi l'émergence de droits protecteurs au bénéfice de l'environnement (2).

\section{L'homme s'inclinant devant la nature : un droit fondamentalement respectueux de l'environnement}

10 La caractère fondamental de la nature commande à l'homme d'agir sur le mode d'un comportement donné. L'éthique de la responsabilité qui sous-tend une attitude déférente à l'égard de la nature entraînera corrélativement l'apparition d'un appareil normatif impliquant une démarche anthropocentrique subordonnée à l'ordre naturel. À des degrés divers, selon qu'on soit placé ici ou là, c'est bien parce que l'homme va s'incliner devant la 
nature que le droit de l'environnement pourra émerger (1.1). Cette manière de voir, a entre autre pour conséquence, la construction d'un édifice juridique qui va consacrer la perpétuation de l'espèce humaine (1.2).

\subsection{La vision de la nature exprimée par les sociétés autochtones et mystiques}

11 La fameuse formule interrogative de Blaise Pascal à propos de l'homme dans la nature issue de son œuvre magistrale posthume les Pensées, rejoint parfaitement la philosophie des peuples autochtones sur le même sujet. Pascal s'interrogeait en ces termes : "Qu'estce que l'homme dans la nature? » Il est loisible d'établir une convergence de vue pour ce qui est une forme de divinisation de la nature. La pensée mystique participe de cette divinisation universelle de la nature (1.1.1) à laquelle on peut adjoindre, voire faire coïncider les convictions des sociétés autochtones (1.1.2).

\subsubsection{Une divinisation universelle de la nature : la pensée mystique}

12 Le mysticisme revêt à l'image de la nature cette dimension universelle en étant présente dans toutes les civilisations qui font de Dieu leur passion. Celui-ci « appartient à l'Inde brahmanique et bouddhiste, à la Chine convertie au culte de Fô et à la doctrine de LaoTseu, à la Grèce païenne lorsqu'elle mêle aux enseignements de Platon les inspirations de l'Orient, à la Judée attentive aux mystères de la Kabbale et aux nations chrétiennes de l'Occident ${ }^{4} »$. Les mystiques croient aux dons de la nature légués aux hommes. Dans leur perception, la nature est une continuation de l'éternités. De ce fait, l'homme ne doit pas s'intéresser uniquement à sa propre évolution mais doit aussi être rendu responsable de l'état de la nature.

13 L'homme est un "extrait du divin universel », faisant de lui une créature de la nature définie comme "une force sensible et intelligente, répandue dans l'univers pour en entretenir le mouvement, la vie ${ }^{6} »$. Cette manière d'envisager la nature fait écho à l'invocation d'une forme de transcendance consubstantielle au divin.

En théologie, la représentation divine est définie comme une sorte "d'intelligence désincarnée qui existe en dehors de la création, dans un espace hors du $\operatorname{cosmos}^{7} »$. Le divin est compris comme étant la source et la puissance de vie, dans, et à travers toutes choses. Il n'est pas séparé de l'ensemble du monde naturel. Cette assertion indique que la séparation du divin d'avec la nature est inconcevable. En liant théologie et philosophie, il est possible d'établir que la nature relevant de l'ordre du divin représente «la grandeur suprême ${ }^{8}$ ".

Le deuxième élément de correspondance réside dans le lien qui existe entre le droit de l'environnement et la finalité du dessein divin : la préservation de l'humanité. Il s'avère en effet que la relation de l'homme avec la nature ou avec son milieu naturel fait appel à «des notions qui impliquent les mythes, les traditions, les religions, les cultures, les systèmes philosophiques et économiques ${ }^{9} »$. S'inspirant de l'évangile, saint Augustin affirme que «le seul Maître de tous est au ciel ${ }^{10}$ ». Étant le propriétaire de toute chose, le "Maître " du ciel fait don aux hommes de la terre pour en tirer la subsistance indispensable à leur vie. Il contribue ainsi de manière décisive à la conservation de l'espèce humaine. 

circumpolaire, les Samis de l'Europe septentrionale, les aborigènes et les insulaires du détroit de Torres d'Australie, et les Maoris de Nouvelle-Zélande. Ces peuples et la plupart des autres peuples autochtones ont conservé des caractéristiques sociales, culturelles, économiques et politiques qui se distinguent nettement de celles des autres groupes qui composent les populations nationales. Les peuples autochtones forment à ce titre ce qu'on peut présenter comme des sociétés traditionnelles.

En Amérique du Sud, la Bolivie, la Colombie, l'Équateur, le Mexique, le Nicaragua, le Paraguay, le Pérou et le Venezuela acceptent le principe de pluralité juridique dans leurs constitutions respectives, en reconnaissant le caractère multiculturel et multiethnique de leurs sociétés. La plupart des peuples autochtones entretiennent une relation particulière avec les terres et les territoires qu'ils occupent. C'est là que leurs ancêtres ont vécu et là que leur histoire, leurs connaissances, leurs modes de subsistance et leurs croyances se sont développés. Pour la plupart des peuples autochtones, le territoire a une valeur sacrée ou spirituelle qui va bien au-delà de l'aspect productif et économique de la terre. transcendance qui doit ipso facto induire le respect de la nature comme vecteur de la survie de l'humanité. Dans ce contexte, le droit devient alors indissociable de la protection de l'environnement parce qu'il est étroitement lié à toute forme de protection par l'interdiction et/ou la prescription de comportement. La volonté de protection dans le domaine de l'environnement est alors subordonnée à des normes juridiques, c'est-àdire des règles obligatoires donc contraignantes. Le recours au droit intervient comme instrument d'encadrement $\mathrm{du}$ comportement des acteurs sociaux vis-à-vis de l'environnement, jouant ainsi un rôle préventif, protecteur, dissuasif et curatif.

de l'environnement qui conserve par conséquent l'idée de droit(s) dévolu(s) à la nature ou encore l'obligation pour l'homme de ne pas la compromettre par ses actions. Le droit de l'environnement est fondé sur le devoir de la protection de la nature, qui lui-même a pour base une référence théiste ${ }^{11}$, sacrée ${ }^{12}$, canonique ou éthique ${ }^{13}$ selon l'analyse défendue. 'homme a besoin d'être convaincu par crainte ou contrainte de la juste place qu'il lui convient d'occuper dans la nature. Qu'il soit croyant ou athée, scientifique ou religieux, philosophe ou dévot, ou encore chamaniste comme dans les sociétés autochtones, l'épineuse question de sa survie par et grâce à la nature est en jeu.

1.1.2. Une constante vision de la divinisation de la nature par les sociétés autochtones

es peuples autochtones vivent dans de vastes régions de la surface de la terre. Disséminés dans l'ensemble du monde de l'Arctique au Pacifique Sud, ils sont, d'après une estimation approximative, quelque 300 millions. Les peuples autochtones ou aborigènes sont ainsi dénommés car ils vivaient sur leurs terres avant que des colons venus d'ailleurs ne s'y installent. Ils sont - selon une définition - les descendants de ceux qui habitaient dans un pays ou une région géographique à l'époque où des groupes de population de cultures ou d'origines ethniques différentes y sont arrivés et sont devenus par la suite 
21 Le rapporteur spécial de l'ONU, M. Martinez Cobo indiquait qu'il est « capital de connaître et de comprendre la relation spéciale, profondément spirituelle, que les peuples indigènes entretiennent avec leur terre, qui est essentielle dans leurs existences, croyances, coutumes, traditions et cultures [...]»: « Pour ces peuples, la terre n'est pas uniquement un bien qu'on possède ou qu'on exploite [...]. Leur terre n'est pas un bien qui peut être acquis, mais un élément matériel dont on doit pouvoir jouir librement ${ }^{14}$. "

«Les peuples autochtones sont enracinés dans des cosmologies complexes où l'interdépendance entre l'homme et la nature apparaît comme une valeur fondamentale, l'équilibre et l'harmonie étant privilégiés au détriment du profit à court terme. » La relation entretenue avec la nature est dénommée "cosmovision ». Le point de départ n'est pas l'individu mais le tout complexe qu'est l'univers entier et la communauté. « Il existe un postulat de départ selon lequel les peuples autochtones se considèrent comme faisant partie de la nature : souvent ils revendiquent le fait d'être inséparables d'elle. Ils sont donc au cœur de la nature : la cosmovision de nombreux peuples se caractérise par l'existence de liens généalogiques entre la nature et ses divers éléments: les êtres humains présentent des liens de parenté avec les autres naturels, vivants ou non ${ }^{15}$. »

23 La dimension mythique y est très prégnante, au sens du mythe défini comme étant à l'origine des règles de régulation sociale, qu'elles soient morales, religieuses ou juridiques. Entre l'homme et la nature, il existe une relation profondément spirituelle, voire religieuse : les peuples autochtones parlent souvent de la nature comme la terre mère. Les êtres humains font ainsi partie d'un tout plus vaste. Les ressources de la nature sont perçues comme des êtres vivants qui méritent respect et protection. La terre n'est qu'une partie du corps social de la nature. La terre n'appartient pas à l'homme. C'est l'homme qui appartient à la terre.

24 Les peuples andins évoquent « la Pachamama » : les divinités sont présentes partout dans la nature. Les montagnes, le soleil, la lune, les étoiles, les formations écologiques particulières, les lacs, etc. comportent une dimension sacrée. La Pachamama représente la pierre angulaire à partir de laquelle on construit les croyances.

25 La nature entretient ainsi de multiples connexions avec les êtres humains. Pour le Conseil autochtone d'Amérique centrale, cette vision du monde est au cœur de la philosophie et de la pensée de l'action collective des peuples autochtones. Cette vision a pour conséquence que les ressources naturelles comme la terre, l'eau, les minéraux, les végétaux ne peuvent être livrés au commerce. Beaucoup de communautés autochtones ritualisent et redistribuent leur surplus, recherchant l'équilibre et l'harmonie ${ }^{16}$. La recherche constante de l'équilibre et de l'harmonie est pour ces sociétés, la base de la perpétuation de l'espèce humaine.

\subsection{La normativisation de la perpétuation de l'espèce humaine}

Chaque organe de la nature joue un rôle donné dans le fonctionnement du métabolisme total qu'est l'univers. L'homme étant un organe de la nature, sa préservation constitue alors un acte cardinal, dans la mesure où la relation d'harmonie ne doit pas être brisée. Issu de l'ordre divin, l'homme doit œuvrer à la perpétuation de son espèce, non dans un esprit anthropocentré visant à le placer au-dessus des autres êtres de la nature, mais dans la perspective de prévenir ce que les mystiques appellent sa chute et qui le conduit inéluctablement à porter atteinte à l'ordre de la nature. Une approche de philosophie 
juridique mise en relation avec le droit de l'environnement(1.2.1) a conduit progressivement à la normativisation de la notion de « générations futures » (1.2.2).

\subsubsection{Une approche de philosophie juridique de la perpétuation de l'espèce humaine} enfin, qu'est-ce l'homme dans la nature ? Un néant à l'égard de l'infini, un tout à l'égard du néant, un milieu entre rien et tout. Il est infiniment éloigné des deux extrêmes ; et son être n'est pas moins distant du néant d'où il est tiré, que de l'infini où il est englouti1 ${ }^{17}$. »

la distance avec le néant évoqué par Blaise Pascal ${ }^{18}$. Dans le même temps il n'est que peu de chose dans la nature, d'où l'idée d'engloutissement qui l'enjoint à cultiver l'humilité face aux choses extrêmes qui le dépassent.

Dans le raisonnement de Pascal, il y a une exigence d'humilité face à la nature, ce qui conduit à subordonner la règle et le comportement humains au(x) droit(s) de la nature qui lui offre l'essentiel, ce dont il a besoin pour vivre. Le caractère infini que les mystiques reconnaissent à la nature les incline à dire qu'elle « n'est pas seulement active, vivante, sensible ; elle est la source de toute activité, de toute vie, de toute sensibilité dans la création ${ }^{19}$ ».

La nature est certainement dans l'esprit des apologistes de la religion chrétienne un des attributs premiers de l'infini dans lequel il y a l'homme, forme incarnée et vivante entre «rien et tout ». La mise en rapport de l'homme avec la nature est le témoignage d'une triple relation. Premièrement, une relation de dépendance plaçant la nature au-dessus de l'homme; une relation d'harmonie, car l'homme est issu de la nature qui l'entretient et enfin, une relation de disharmonie dans la mesure où l'homme peut être un élément nuisible pour la nature.

31 La pensée mystique ne croit pas à la bonté originelle de l'homme agissant sous l'empire des lois de la nature comme le pensait Rousseau ${ }^{20}$ et ses inspirateurs. Il lui préfère la théorie de la chute : «l'homme est un esprit tombé de l'ordre divin dans l'état naturel et qui tend à remonter à son premier état. " Il est ainsi à l'origine "des désordres de l'univers et de la sociétéé ${ }^{11}$.

Il est possible de rapprocher cette façon de voir de celle qui consiste à expliquer l'origine du droit de l'environnement dans la "collision entre l'histoire naturelle et l'histoire humaine ${ }^{22}$ ». Il est difficile de contester les effets parfois négatifs de l'action anthropique qui vient bousculer l'ordre naturel établi parce que celle-ci fait fi du droit à la préservation de l'intégrité de l'environnement. L'homme, en se rendant coupable d'atteinte à l'environnement appelle la création d'un droit qui sera autant protecteur de la nature que coercitif, et ce au bénéfice des générations futures.

\subsubsection{La normativisation de la notion de " générations futures "}

De manière sous-jacente et incidente, lorsque l'on fait référence au futur en lien avec des personnes, cela consiste à penser qu'elles ont droit à la vie, qu'elles ont vocation à accéder à la vie. Et comment peuvent-elles bénéficier du statut du droit à la vie, si la transmission de la vie est compromise? L'héritage est impossible lorsque l'objet en question, matériel ou immatériel, est annihilé. 

l'avenir dans les sociétés autochtones. La transmission fonde la conservation des valeurs, des attitudes et des aptitudes nécessaires à la perpétuation du groupe. Appliqué au droit de la protection de l'environnement, celui-ci apparaît comme une courroie de transmission dans la chaîne de la perpétuation de la vie, un vecteur de la garantie du bien être qui permettra à l'homme d'assurer sa descendance. L'ontologie autochtone repose sur une relation harmonieuse et respectueuse avec le territoire et la terre mère. accéder à la vie procède d'une éthique écologique. La pensée autochtone sert de recours si comme l'affirme Erich Fromm, il y a en Occident « une passion de détruire ", inclinant à étudier « l'anatomie de la destructivité humaine ${ }^{23}$ ».

L'alternative de Fromm consiste à choisir pour humanité globalisée «des valeurs humanistes, qui peuvent être revitalisées et actualisées en intégrant les valeurs des autres traditions culturelles du patrimoine de l'humanité, traditions qui ont toujours consciemment gardé le champ économique sous l'autorité du champ social, et qui ont, mieux que l'Occident, intégré la relation symbiotique avec la nature dans leur cosmogonie, leur organisation sociale et culturelle ${ }^{24} »$.

37 Alors que la pensée philosophique des sociétés autochtones s'appuie sur les notions d'équilibre, d'harmonie et de symbiose, l'Occident moderne se fonde sur l'opposition généralisée: raison-passion, état de nature-culture, écologie-industrie, progrèsprimitivisme, nature-homme.

38 La spiritualité autochtone est très proche de la nature. L'animisme, de animus, esprit ou âme, pose comme principe que tous les êtres vivants, hommes, animaux et végétaux, mais aussi les éléments naturels, comme les minéraux, l'eau, le vent ou le feu, participent de la force vitale de l'univers et, à ce titre, possèdent une âme. En fait, «l'animisme recouvre deux dogmes : la croyance à l'âme et à une vie future et, corrélativement, la croyance à des divinités directrices et à des esprits subordonnés ${ }^{25}$ ».

39 Le dogme de la vie future dans la pensée autochtone fait écho à la notion de " générations futures » qui apparaît pour la première fois en 1968, à l'occasion de la création du club de Rome lorsqu'émerge l'idée de développement durable. Pour la célèbre organisation non gouvernementale World Wildlife Fund (WWF), le rôle que doivent jouer les groupes autochtones dans le succès du dessein de la préservation de l'espèce humaine ne fait aucun doute.

Qu'on en juge par le contenu du premier alinéa du préambule des Principes pour la coopération entre le WWF et les organisations des peuples autochtones pour la conservation de la biodiversité sur leurs terres et territoires et pour la promotion de l'utilisation durable des ressources naturelles adoptés le 27 juin 1989 : «Les peuples autochtones vivent dans la plupart des régions importantes de grande valeur naturelle qui subsistent encore. Ceci démontre l'efficacité des systèmes indigènes et tribaux de gestion des ressources. Les peuples autochtones et les organisations de conservation devraient donc être des alliés naturels dans la lutte pour la conservation d'un monde naturel sain et de sociétés humaines saines. Malheureusement, les objectifs de la conservation de la biodiversité d'une part, de la protection et de la préservation des cultures et des moyens de subsistance des peuples autochtones d'autre part, ont parfois été perçus comme étant contradictoires plutôt que comme deux intentions qui s'appuient mutuellement ${ }^{26}$.» 
41 L'homme en s'inclinant devant la nature démontre sa volonté d'être respectueux de l'environnement en organisant entre autre des droits protecteurs au bénéfice de l'environnement.

\section{L'homme s'inclinant devant la nature : le développement de droits protecteurs au bénéfice de l'environnement}

42 L'homme n'est pas le seul être vivant de l'ordre naturel. Pour autant, son comportement laisserait à penser qu'il en est ainsi. L'action anthropique peut générer bien des effets désastreux sur la nature et les autres êtres vivants qui la composent. Ceux-là même, qui à bien des égards, constituent les conditions indispensables de sa subsistance, voire de sa perpétuation en tant qu'espèce. Aujourd'hui, il est de plus en plus question de crime contre l'environnement, le criminel désigné étant l'homme.

Dans une posture tout à fait inversée, on distingue l'homme déférent à l'endroit de la nature. Il est autant présent dans la philosophie des sociétés autochtones que dans la pensée mystique. Ces derniers évoquant pour leur part, «l'homme divin», «l'homme esprit », «l'homme réparé27 ». Dans ce contexte, la chosification de la nature (nature objet) sera remplacée par une vision d'humanisation (nature sujet), voire de déification de la nature. À partir des deux points de vue, mystique et autochtone, il est possible d'énoncer un droit de l'environnement humaniste (2.1), de même qu'est envisagée la question des droits conférés à la nature (2.2).

\subsection{Un droit de l'environnement d'essence humaniste}

44 Une approche humaniste de l'environnement développée par les sociétés chrétiennes mystiques et les sociétés autochtones a un point de jonction essentiel, celui d'une asymétrie entre l'homme et la nature. La nature étant placée au-dessus de l'homme dans leur philosophie. De plus, toutes les autres créatures de la nature ne sont pas dépourvues de droits, à tout le moins, elles n'en ont pas moins que les hommes. Très récemment, l'Assemblée nationale a reconnu aux animaux la qualité symbolique «d'êtres vivants doués de sensibilité28 ${ }^{2}$.

45 La philosophie mystique et chrétienne de la nature fait référence à Dieu (2.1.1), alors que celle des civilisations autochtones privilégie la dimension cosmogonique (2.1.2).

\subsubsection{L'approche mystique : la coïncidence entre Dieu et la nature}

"Si tu déifies seulement l'intelligence et la science, pouvoirs suprêmes du moi hautain, tu t'es déjà donné au diable, et avec lui tu périras ${ }^{29}$.» Ainsi s'exprime le point de vue mystique et apologétique de la religion chrétienne qui ne partage pas la vision du jus naturalis. Selon cette pensée juridique, le cosmologisme antique et le théologisme médiéval doivent être congédiés. La nature, œuvre d'un dieu créateur et organisateur se trouvera reléguée à un rang subalterne. Grotius, mathématicien comme Pascal déconnecte la création du droit en vigueur de l'existence de Dieu. Pour le juriste hollandais Grotius (1583-1645), la validité du droit naturel ne repose pas sur un quelconque commandement divin, mais bel et bien sur la nature sociale de l'homme ${ }^{30}$. 
Pour autant, Grotius pourrait rejoindre Pascal sur le fait que l'ordre normatif (légal et moral) transcende la volonté ou l'autorité des acteurs ${ }^{31}$. La convergence résiderait dans un ordre moral placé au-dessus, guidant la volonté des faiseurs de la norme.

L'ordre normatif moral dans lequel peuvent se reconnaître les mystiques rapproche l'humanité de "l'homme divin » ou de «l'homme réparé », servant par la même occasion un droit de l'environnement protecteur. Le sort réservé au(x) droit(s) de la nature et l'essor pris ou non par le droit de l'environnement dépendent de la capacité de l'homme à stopper sa « chute » en s'astreignant à respecter l'ordre normatif moral transcendant. Le théocentrisme s'oppose à l'anthropocentrisme axé sur la raison qui va parfois jusqu'à discréditer et à exclure les plus hautes formes de la contemplation mystique.

« Le droit naturel est une règle qui nous est suggérée par la droite raison d'après laquelle nous jugeons nécessairement qu'une action est injuste ou morale selon sa conformité avec la nature raisonnable et qu'ainsi Dieu qui est l'auteur de la nature défend l'une et commande l'autre ${ }^{32}$. » Cette définition ramène sensiblement la notion de droit naturel à la notion de morale. Toutefois, la référence de Grotius n'est pas seulement d'ordre théologique, mais aussi d'ordre logique. En effet, Grotius déclare dans le même temps: "Nous devrions admettre le droit naturel même si l'on concédait - concession criminelle - que Dieu n'existe pas $^{33}$.» La thèse de notre auteur consiste donc à représenter le droit naturel comme une forme de la vérité éternelle répandue dans l'univers, perceptible à la raison humaine de la même manière que les axiomes de la géométrie.

Les premiers juristes de droit naturel tout en concédant la place de choix qui doit être octroyée à la raison humaine, ne reconnaissent pas moins l'existence de Dieu, car une autre attitude serait « une concession criminelle » dit Grotius. De même, s'il est logique que l'homme puisse exploiter les fruits de la nature pour assurer sa subsistance, une approche morale l'enjoint à en user avec sagesse. La revendication d'une éthique de la nature favorise la mise en place d'un ordre normatif écologique auquel «l'homme esprit » se soumet, échappant ainsi à sa condition « d'homme animal ».

$\mathrm{Au} \mathrm{XVII}^{\mathrm{e}}$ siècle, la réaction chrétienne contre la valorisation de l'homme dont la Renaissance est le terreau fertile, vise à rejeter l'anthropocentrisme qui en découle. L'homme n'est rien en face de l'immensité de Dieu qui est révélé par la nature, la logique, mais surtout par la foi, don divin, don surnaturel: «La foi dit bien ce que les sens ne disent pas, mais non pas le contraire de ce qu'ils voient; elle est au-dessus, et non pas contre $^{34}$.»

\subsubsection{L'approche des civilisations autochtones : la nature vécue à travers le prisme de la cosmogonie}

La conception d'un droit de l'environnement inspirée de considération morale est imprégnée d'une certaine dimension théocratique qui comporte des racines universelles.

En effet, on observe que « les écologistes bouddhistes étendent l'amour et la compassion au-delà des personnes et des animaux en incluant les plantes et la terre elle-même ${ }^{35}$ ». Les hindouistes pour leur part considèrent que la nature doit être respectée parce qu'elle incarne dans ses composantes les manifestations de la divinité et des esprits à différents stades d'évolution spirituelle ${ }^{36}$. 
Le taoïsme, religion dominante en Chine à côté du confucianisme, part du principe que l'homme ne doit pas manifester la prétention à la domination de la nature, mais au contraire doit s'insérer dans l'ordre universel et chercher l'équilibre dans la nature. Le confucianisme considère, de son côté, l'homme comme produit de la nature. Les conceptions confucianiste et taoïste se rapprochent des religions appartenant à la catégorie du naturisme, telles que l'animisme, le totémisme, le chamanisme, etc.

Enfin, les religions monothéistes, dites révélées que sont le judaïsme, le christianisme et l'islam sont davantage anthropocentrées, ce qui ne signifie pas pour autant une exploitation irraisonnée des biens de la nature, rendant alors possible un droit «à » et « de » l'environnement.

S'appuyant sur la pensée des sociétés autochtones, certains auteurs, pour vivifier le droit à l'environnement préconisent un retour à la valeur spirituelle de la nature qui a été détruite. Il s'agit par conséquent de redécouvrir l'essence sacrée de la nature. Au travers d'un postulat traditionnel, le rapport à la nature est fondé sur la transcendance relevant d'une adhésion à une démarche spirituelle élevée.

6 La vision des Amérindiens procède de cette démarche. Leur foi en la nature fait corps avec une tradition métaphysique, en tant que forme supérieure de connaissance englobant tous les savoirs et tous les phénomènes, dans le cadre d'une cosmogonie qui fonde leur identité.

7 Il est intéressant d'observer que certains penseurs du début de la chrétienté, mystiques et théologiens, comme maître Eckhart ${ }^{37}$, étaient métaphysiciens. Dans l'histoire de l'islam et dans la civilisation chinoise, il est possible de discerner une connexion intime entre la dimension métaphysique de la tradition et l'étude de la nature. Dans ces deux géographies philosophiques, l'observation de la nature était le fait des côtés gnostique et mystique de la tradition, formant le lien indivisible entre les humains et la nature.

En refusant de séparer complètement l'homme et la nature, les sociétés autochtones cherchent à préserver une vision intégrale de l'univers, en voyant dans les artères de l'ordre cosmique et naturel du monde le flux de la grâce divine; cette grâce divine qualifiée par les musulmans de «barakah » et par les chrétiens de " providence ».

Aujourd'hui, les luttes conduites par les sociétés autochtones devant les juridictions internationales pour faire valoir leur vision identitaire et spirituelle concourent à transformer la perception que peut avoir une société industrialisée de la nature.

Des juges de l'hémisphère américain estiment que « les relations des indigènes à la terre [ne sont] pas seulement une question de possession et de production, mais un élément matériel et spirituel dont ils doivent jouir pleinement, même pour préserver leur héritage culturel et de transmettre aux générations futures»; que « la culture des membres des communautés autochtones correspond à un mode de vie particulier d'être, de voir et d'agir dans le monde, formé à partir de sa relation étroite avec leurs terres traditionnelles et des ressources qui s'y trouvent... Elles constituent une partie intégrante de leur vision du monde, de la religion et, par conséquent, de leur identité culturelle ${ }^{38}$.»

La protection et la préservation de l'identité spirituelle des peuples autochtones sert la cause de l'éclosion d'une pensée juridique qui soutient l'octroi de droits au bénéfice de la nature. 


\subsection{Vers des droits conférés à la nature ?}

62 Dans sa relation avec la nature, l'homme se trouve fréquemment dans une situation d'abus de droits, notoirement dans les États industrialisés, nouvellement industrialisés et en voie d'industrialisation. La notion de croissance économique qui fait cadencer le cœur de ces sociétés a pour fondement philosophique l'obsession de la quantité par l'accumulation du capital et des biens.

Dans une pareille configuration, nous sommes loin des civilisations qui accordent une âme à tout être et à toute chose, assortie de la possibilité d'une communication interactive entre eux. La relation fusionnelle et spirituelle de l'homme à la nature implique que si l'homme est titulaire de droits, il ne peut en être autrement de la nature (2.2.1). Les chrétiens mystiques ainsi que les civilisations autochtones en sont convaincus. Reste à savoir quelle est l'influence exercée par leur conception sur le droit de l'environnement moderne (2.2.2).

\subsubsection{Quelle pertinence d'une titularité de droits au profit de la nature?}

Dans le contexte de l'homme conscient, celui-ci n'a aucun mal à concéder qu'une plante ou un animal n'est pas simplement un bien meuble. Ces êtres vivants sont aussi titulaires de droits que les humains ont l'obligation de respecter.

La philosophie en se muant en écosophie vise le biocentrisme (l'effort d'étendre la valeur morale à toute forme de vie ${ }^{39}$ ). L'écosophie est aussi en lien avec l'écocentrisme, qui par opposition à l'éthique biocentrique de l'environnement correspond à un élargissement du domaine moral jusqu'aux éléments non vivants de la nature. Il est question d'un égalitarisme biosphérique (biospheric egalitarianism) qui aboutit à la protection dans leur intégrité des communautés, des écosystèmes. L'homme fait partie de la communauté biotique, les plantes et les animaux sont ses compagnons, d'où la nécessité d'un partenariat (stewardship) avec la nature.

Dans la philosophie des sociétés autochtones et des mystiques, l'affirmation de droits conférés à la nature est subordonnée à une posture anthropique de déférence vis-à-vis de la nature. À la vérité, pour ces confessions de pensée, l'idée même de droits conférés à la nature est un non-sens puisque l'homme est produit et dépendant de la nature.

L'homme au cœur gonflé d'orgueil va s'ériger en maître de l'ordre naturel. À l'inverse, les mystiques, au travers de Blaise Pascal, nous enseignent que bien qu'il soit un roseau pensant, l'homme dans la nature est un néant à l'égard de l'infini. Dans la pensée mystique et dans les sociétés traditionnelles la valeur comportementale à transmettre pour ce qui concerne l'espèce humaine, relève de «l'homme divin » ou « réparé »; chez les autochtones, l'homme protecteur et respectueux de la terre mère.

68 Paul Ricoeur ${ }^{40}$ se plaît à souligner ce que disait Kant à propos de l'homme : cet animal qui est le moins bien équipé pour survivre. Paul Ricoeur ajoute en citant cette fois Hans Jonas que ces projets de maîtrise de la nature aboutissent à l'autodestruction. «Il n'est pas évident que l'humanité survive ", conclût-il.

«Les visions classiques de l'histoire réservent à l'homme la place privilégiée - l'histoire était faite de ses idées, ses conceptions, ses actes de courage, ses conquêtes etc. L'environnement était souvent en second plan. Cette attitude des historiens, philosophes, écrivains de la période moderne est imprégnée de la vision mécaniciste du monde, née 
avec Newton, Descartes et Bacon et prolongée jusqu'au $\mathrm{xx}^{\mathrm{e}}$ siècle ${ }^{41}$. Au regard d'une conception constamment anthropocentrée, quelles sont la place et l'influence de la pensée mystique et des autochtones sur le droit moderne de l'environnement?

\subsubsection{Quelle influence de la pensée mystique et des civilisations autochtones sur le droit de l'environnement?}

Pour Maurice Kamto, l'interdisciplinarité et le divin, y compris ses aspects périphériques (religion, mythes, traditions etc.) sont contenus dans la définition du droit de l'environnement livrée par la doctrine.

71 À propos de l'interdisciplinarité, il est permis d'observer que « l'environnement constitue un nouveau champ de la recherche sans frontières ». « Après les développements récents du droit de la mer, l'environnement remet en effet l'interdisciplinarité à l'honneur. Parce qu'il englobe tous les éléments de la nature reliés par des rapports d'interdépendance systématiques, l'environnement ignore les murs de souveraineté érigés par les États. Parce qu'il se conçoit à la fois comme connaissance du milieu naturel et protection dudit milieu, il transcende les frontières disciplinaires qui ceinturent les spécialistes et transforment le savoir scientifique en une tour de Babel. L'environnement restaure le dialogue des sciences en mettant à contribution divers champs du savoir pour relever un seul et même défi : celui de la survie de l'humanitét2."

72 À propos de ses aspects périphériques (mythes, religions, traditions), l'éthique de l'environnement est invocable d'après ce que nous rapporte Cicu-Gabriel Arsène lorsqu'il expose que «la relation de l'homme avec l'environnement/la nature, ou plutôt les relations de l'humanité avec son milieu naturel, constitue un sujet dont le traitement, même sommaire, requiert des notions qui impliquent le mythe, les traditions, les religions, les cultures, les systèmes philosophiques, politiques et économiques ${ }^{43}$ ».

Dans le monde occidental gagné par le progrès technique et les prouesses technologiques, l'avènement du monde-machine a progressivement, à partir du XVII siècle, procédé à l'éviction de la foi au profit de la raison. La nature n'est pas pensée au travers d'une forme de transcendance, mais à partir de l'homme doté du logos et de la raison. De ce fait, l'univers, le monde et la nature ne sont que des objets d'étude (et d'exploitation) dont les secrets méritent d'être dévoilés.

Dans le même temps, d'autres civilisations continuent de perpétuer un rapport à la terre qui remonte à des temps immémoriaux, intimement convaincues que l'asymétrie hommenature ne confère pas la prééminence au premier. Les sociétés autochtones autant que les mystiques parviennent à la conclusion que l'éloignement de l'homme de la nature le conduit à avoir un cœur dur ; l'oubli du respect dû à ce qui pousse et à ce qui vit amène également à ne plus respecter l'homme ${ }^{44}$. L'homme s'illustre alors comme un loup pour l'homme, comme un loup pour la nature.

Michel Prieur ${ }^{45}$ défend l'idée d'un droit de l'environnement qui s'apparente à ce que Kant appelait le droit cosmopolitique. Il y a désormais des principes communs aux peuples de la planète, expression d'une solidarité mondiale due à la globalité des problèmes d'environnement. Ainsi la déclaration de Rio de 1992 évoque « un partenariat mondial sur une base nouvelle», en reconnaissant que «la terre, foyer de l'humanité, constitue un tout marqué par l'interdépendance ». 
76 Le droit de l'environnement de nature cosmopolitique signifie qu'il se nourrit des apport de toutes les contributions sociales mondiales, y compris les sociétés autochtones. À cet égard, le principe de précaution fournit l'illustration de «l'homme réparé » (le retour au divin) ; celui qui s'incline et manifeste de la déférence vis-à-vis de la nature. En effet, en revendiquant un droit à l'environnement préservé et sain défendu, est affirmé un droit de l'environnement assorti de principes cardinaux tels le principe fondamental de précaution ${ }^{46}$.

77 Le principe de précaution peut certes faire l'objet d'une glose très dense de la part de techniciens et d'exégètes du droit, mais en même temps, on pourrait en extraire une part de substance d'essence théologique, en ce qu'il consacre la magnificence de la transcendance que l'on retrouve à la fois chez les mystiques et dans le contenu du discours sur la nature tenu par les membres des sociétés autochtones.

Le principe de précaution semble exprimer la pleine conscience de "l'homme esprit » évoqué par les mystiques, relativisant ainsi la prétendue toute puissance de la raison ${ }^{47}$. $\mathrm{Ce}$ peut être la manifestation de "l'homme divin» ou de "l'homme réparé » qui fait allégeance à la nature, incliné à respecter ce qu'il ignore, ce qui le dépasse. La convention des Nations unies sur la diversité biologique de 1992 s'inscrit à cet égard dans cette ligne, en indiquant que «la diversité biologique s'appauvrit considérablement par suite de certaines des activités de 1 'homme ${ }^{48} »$.

En outre, lorsqu'il existe une menace de réduction sensible ou de perte de la diversité biologique, l'absence de certitudes scientifiques totales ne doit pas être invoquée comme raison pour différer les mesures qui permettraient d'en éviter le danger ou d'en atténuer les effets.

Enfin, la convention note "qu'un grand nombre de communautés locales et de populations autochtones dépendent étroitement et traditionnellement des ressources biologiques sur lesquelles sont fondées leurs traditions et qu'il est souhaitable d'assurer le partage équitable des avantages découlant de l'utilisation des connaissances, innovations et pratiques traditionnelles intéressant la conservation de la diversité biologique et l'utilisation durable de ses éléments ${ }^{49} »$.

\section{Conclusion}

81 «Pour la première fois dans l'histoire de l'humanité, les actions de l'homme pourraient se révéler irréversibles. Or, en face, c'est le "vide éthique", car les morales traditionnelles sont trop courtes. Elles sont particulièrement inopérantes pour les décideurs politiques ${ }^{50}$ .» Tel est le propos tenu par Hans Jonas dans son célèbre ouvrage Le Principe responsabilité. De cette observation du philosophe, on notera avec intérêt la mention du recours aux morales traditionnelles enracinées dans la conviction mystique, détenues et pratiquées aujourd'hui encore par les sociétés autochtones. Si le droit de l'environnement est un outil au service de la protection et de la préservation de la nature, comment pourrait-on occulter l'héritage universel de la philosophie mystique, de même que le riche patrimoine des savoirs des sociétés autochtones? 


\section{NOTES}

1. Trinh Xuan Thuan, Le Cosmos et le lotus, Paris, Albin Michel, 2011, 258 p.

2. «Le droit naturel ou droit de nature signifie qui découle de la nature. On peut l'entendre dans deux sens : le droit qui retrouve dans la nature son fondement, ou le droit qui reçoit de la nature sa justification. L'ordre naturel est en conséquence l'ordre des choses de la Création. Par naturel, il ne faut pas entendre tout ce qui existe dans la nature (ou dans la réalité sociale), mais tout ce qui est conforme à la nature des choses » (Mohammad Javad Javid, Droit naturel et droit divin comme fondements de la légitimité politique: une étude comparative du christianisme et de l'Islam, thèse de doctorat, Henry Roussillon (dir.), université des sciences sociales de Toulouse, 2005, p. 27).

3. «[...] le dialogue des sources à la recherche de la protection la plus favorable aux victimes et révolutionnant ainsi la portée du droit à l'environnement. Ce dernier progresse vers un processus de fondamentalisation dans les situations conflictuelles où les populations autochtones vulnérables opposent leurs droits à l'exploitation des ressources naturelles accaparées par les États, en raison du développement économique. En ce sens, le système traite des enjeux spécifiques de la réalité américaine, comme par exemple en allant au-delà de la notion occidentale du droit à la propriété, à travers la définition de la propriété communale liée à la nature qui maintient la vie digne des populations concernées; ou créant le dommage spirituel qui trouve aussi des fondements dans la relation des indigènes avec la nature » (Camila Perruso, " La question de la nature dans le système interaméricain des Droits de l'homme : une révolution?", dans Pauline Milon (dir.), Révolution scientifique, révolution juridique: vers une fondamentalisation du droit de l'environnement ?, Presses universitaires d'Aix-Marseille, 2014).

4. Adolphe Franck, La Philosophie mystique en France à la fin du XVIII siècle, Paris, GermerBaillière, 1866, p. 9.

5. Louis-Claude de Saint-Martin, Le Ministère de l'homme-esprit, réédition brochée, Diffusion rosicrucienne, 1999 (édition originale, 1802, Paris, imprimerie de Migneret), p. 84.

6. Adolphe Franck, op. cit., p. 150.

7. Rosemary Radford Ruether, «Le Dieu des possibilités : l'immanence et la transcendance repensée ", Théologiques, vol. 8, n² 2, 2000, p. 35-48, spéc. p. 36.

8. Isabelle Bochet, «Transcendance divine et paradoxe de la foi chrétienne. La polémique de Tertullien contre Marcion ", Recherches de science religieuse, $\mathrm{n}^{\circ}$ 2, 2008, p. 255-274.

9. Gicu-Gabriel Arsène, «La relation homme-nature. L'émergence de l'éthique de l'environnement » : http://bioethics.agrocampus-ouest.eu/infoglueDeliverLive/ digitalAssets/57434_51FR_relation_homme_nature.pdf (consulté le 24 novembre 2014), p. 1.

10. Saint Augustin, De Magistro, 14-46.

11. Emmanuel Goyon, «Droits de l'homme et environnement: la position de l'église catholique ", dans Jérôme Ferrand et Hugues Petit (dir.), L'Odyssée des droits de l'homme, Paris, L'Harmattan, 2003, t. III. 
12. Voir : Michel Serres, Le Contrat naturel, Paris, Flammarion, 1992, $191 \mathrm{p}$.

13. Marie Geneviève Pinsart, Jonas et la liberté : dimensions théologiques, ontologiques, éthiques et politiques, Paris, Vrin, 2001, p. 233 et suiv. ; Joseph Mbungu Mutu, Éthique écologique et principe de la responsabilité, Francfort-sur-le-Main, Lang, 2010, 407 p.

14. José R. Martínez Cobo, Study on the Problem of Discrimination Against Indigenous Populations, Add. 1, § 196-197, Genève, UN Commission on Human Rights, 1986, 276 p. (Sous-commission de la lutte contre les mesures discriminatoires et de la protection des minorités).

15. Frédéric Deroche, Les Peuples autochtones et leur relation originale à la terre: un questionnement pour l'ordre mondial, Paris, L'Harmattan, 2008, p. 40 et 42.

16. Groupe international de travail pour les peuples autochtones (GITPA), Développement et droit coutumier, Paris, L'Harmattan, 2012, p. 91 et suiv.

17. Voir: Blaise Pascal, Pensées, 1670: http://www.philo5.com/Les\%20philosophes\% 20Textes/Pascal_Pensees-HommeRoseau_PariSurDieu.htm (consulté le 24 novembre 2014).

18. Voir : Salvaggio Giuseppe, L'homme dans l'Univers infini de Bruno, TheBookEdition, 2014, 286 p. (mémoire de maîtrise, Louvain-la-Neuve, université catholique de Louvain, Institut supérieur de philosophie, 1992, 235 p.)

19. Adolphe Franck, op. cit., p. 153.

20. «L'homme est bon; c'est la société qui le corrompt.»

21. Adolphe Franck, op. cit., p. 103.

22. Jean Chesneaux, «Maîtriser la collision entre l'histoire naturelle et l'histoire humaine ", Écologie politique, n 2, 1992, p. 127.

23. Erich Fromm, La Passion de détruire: anatomie de la destructivité humaine, trad. Léo Carlier, Paris, Robert Laffont, 2001, 524 p.

24. Léon Grimard, LeXXI siècle: pour une anthropologie de l'écologie: http:// www.academia.edu/2320965/Le_XXIe_si\%C3\%A8cle_pour_une_anthropologie_de_l\%C3\% A9cologie (consulté le 24 novembre 2014).

25. Guy Le Moal, dans Pierre Bonte et Michel Izard (dir.), Dictionnaire de l'ethnologie et de l'anthropologie, Paris, PUF, 2004, p. 72.

26. Pour consulter l'ensemble voir: http://lucy.ukc.ac.uk/rainforest/indigfr.html (consulté le 24 novembre 2014).

27. Voir, par exemple, Blaise Pascal, Pensées, « Disproportion de l'homme », 199-752 H.

28. Entretien avec le juriste Jean-Marc Neumann, Le Monde.fr, 16 avril 2014 : http:// abonnes.lemonde.fr/planete/article/2014/04/16/les-animaux-reconnus-comme-desetres-sensibles-un-pas-totalement-symbolique_4402541_3244.html (consulté le 24 novembre 2014). Voir, également : Assemblée nationale, «Proposition de loi visant à établir la cohérence des textes en accordant un statut juridique particulier à l'animal ", 29 avril 2014, http://www.assemblee-nationale.fr/14/pdf/propositions/pion1903.pdf (consulté le 24 novembre 2014).

29. Franz Xavier von Baader, Les Enseignements secrets de Martinès de Pasqually, Paris, Bibliothèque Chacornac, 1900, p. 3.

30. Jean-François Thibault, « Lecture de Grotius », Politique et sociétés, vol. 19, n 1, 2000, p. 168. 
31. Benedict Kingsbury, «Grotius, Law, and Moral Scepticism: Theory and Practice in the Thought of Hedley Bull », dans Ian Clark et Iver B. Neumann (dir.), Classical Theories of International Relations, New York, St. Martin's Press, 1996, p. 42-70.

32. Cité par Antoine Rougier, «La reconnaissance du droit naturel », Conférence à la société académique vaudoise, 1913, http://livres-mystiques.com/partieTEXTES/Rougier/ renaissa.html (consulté le 24 novembre 2014).

33. Cité par Antoine Rougier, art. cité.

34. Blaise Pascal, Pensées, 185-265.

35. Pascal Koba, Notes de cours des philosophies orientales, Scolasticat Jean XXIII, inéd., Kolwesi, 1998-1999, p. 24.

36. Gicu-Gabriel Arsène, art. cité, p. 6.

37. Voir : Julie Casteigt, Connaissances et vérité chez maître Eckhart, Paris, Vrin, 2006, 480 p.

38. Corte IDH, Caso comunidad indígena Yakye Axa vs. Paraguay, Fondo reparaciones y costas, sentencia de 17 de junio de 2005, serie $C n^{\circ} 125$, párr. 135 y sentencia de 29 de marzo de 2006, serie $C n^{\circ} 146$, párr. 118.

39. «C'est ainsi que E. Bourgeois peut dire qu'il existe un lien étroit de dépendance qui relie l'homme à l'environnement qui l'entoure et, par conséquent, nous ne pouvons nier que ses intérêts soient étroitement liés à ceux du monde physique " (Martino Amisi, Les Rapports entre l'homme et la nature. Une analyse critique de l'éthique de l'environnement, Institut facultaire Théophile Reyn, graduat en philosophie, 2009: http:// www.memoireonline.com/09/09/2716/m_Les-rapports-entre-lhomme-et-la-nature-Unanalyse-critique-de-lEthique-de-lenvironnement9.html (consulté le 24 novembre 2014).

40. «L'éthique, le politique, l'écologie. Entretien avec Paul Ricoeur » (Propos recueillis par Édith et Jean-Paul Deléage, Écologie politique, nº 7, 1993, p. 2).

41. Gicu-Gabriel Arsène, art. cité, p. 2.

42. Maurice Kamto, Droit de l'environnement en Afrique, Vanves, EDICEF, 1996, p. 20.

43. Gicu-Gabriel Arsène, art. cité, p. 2.

44. Teresa Carolyn McLuhan, Pieds nus sur la terre sacrée, Michel Barthélemy (trad.), Paris, Denoël, 1974, p. 14.

45. Michel Prieur, "Les principes généraux du droit de l'environnement », Cours de droit international et comparé de l'environnement, Campus numérique, Envidroit, p. 2 : http:// foad.refer.org/IMG/pdf/Module_5.pdf (consulté le 24 novembre 2014).

46. En réalité le principe de précaution est aussi vieux que le monde. Il est l'expression de la sagesse populaire qui, face à des incertitudes, préfère la prudence à une audace qui pourrait être suicidaire. Il est le refus d'une société ou l'homme jouerait, sans contrôles, à l'apprenti sorcier. Ce principe eut d'ailleurs été mieux qualifié de "principe de prudence » par Michel Prieur («Le principe de précaution», Les $\mathrm{X}^{\mathrm{e}}$ journées juridiques franco-chinoises sur le droit de l'environnement, Paris, 11-19 octobre 2006: http:// www.legiscompare.fr/site-web/IMG/pdf/2-Prieur.pdf, consulté le 24 novembre 2014, p. 1) ; voir également Maryse Deguergue, «Les avancées du principe de précaution en droit administratif français », RID comp. 2006, n² 2, p. 621-641.

47. «Nous voici donc entrés, bien malgré nous, dans l'ère de la précaution. À y bien réfléchir, cette mésaventure est normale. Car Descartes nous avait octroyé des droits sur la nature, sans contrepartie. Or ce qui fait l'intérêt, sinon la noblesse du Droit, c'est 
d'imposer des devoirs corollaires aux droits. C'était la pièce manquante dans les rapports de l'homme et de la Nature. L'organique, si on le viole, ne pardonne pas, dit Teilhard de Chardin.» Et d'ajouter: "Tandis qu'avec le juridique, on arrive toujours à s'arranger... » Pour Teilhard, convaincu de l'unité organistique du monde, l'accroissement du rayon d'action humain implique un renouvellement de l'éthique à la mesure des possibilités technologiques. Hans Jonas, philosophe allemand nourri d'Heidegger et de communisme, donc en principe situé aux antipodes du destin, partage la même intuition. Il nous dit l'interdépendance entre l'homme et la nature : l'intégrité de la nature autour de nous, c'est l'intégrité de la nature en nous; les agressions qui affectent l'une se répercutent inéluctablement sur l'autre. Et il plaide pour une éthique nouvelle élargie dans le temps et dans l'espace, à la mesure des temps qui viennent. Cette éthique élargie s'appelle le "principe de précaution ». Celui-ci comporte notamment une prise en compte de la durée. À la suite de Teilhard et de Jonas il faut désormais que notre société et que son droit apprennent à se projeter dans le futur, à ménager les réversibilités et à éviter l'irréparable au nom des générations futures, voir : Martine Rémond-Gouilloud, «L'ère de la précaution », Revue Archimède et Léonard, « hors-série », n 10, 1993-1994, p. 63.

48. "Préambule ", Convention des Nations unies sur la diversité biologique, 1992, p. 1 : https://www.cbd.int/doc/legal/cbd-fr.pdf (consulté le 24 novembre 2014).

49. «Préambule ", Convention des Nations unies sur la diversité biologique, 1992, op. cit., p. 2.

50. Hans Jonas, Le Principe responsabilité: une éthique pour la civilisation technologique, Paris, Flammarion, 1998, $450 \mathrm{p}$.

\section{RÉSUMÉS}

Il est des régulations sociales par le droit qui sont intrinsèquement universelles. Tel est le cas du droit de l'environnement qui peut être présenté comme l'ensemble des règles et des décisions rendues, mais aussi des pratiques coutumières ayant un rapport avec la nature. Le droit positif de l'environnement est à coup sûr celui que l'on trouve dans les codes ainsi que dans tous les textes normatifs écrits. S'il est important de le considérer, notamment pour des raisons d'efficacité et d'utilité à l'application d'un droit protecteur de l'environnement, il est tout aussi intéressant d'en rechercher les racines. On en arrive à l'art qui consiste à penser le droit, à conceptualiser. La conceptualisation rejoint la fondamentalisation du droit de l'environnement, entendue comme un processus qui tend à constituer les bases, l'assise de la matière. Les concepts constituent ainsi les composantes d'une matière juridique évoluée devenue système. Elles sont aussi porteuses de valeurs et révèlent ce qui fait l'essence même de ce droit.

Some legally encoded social rules are intrinsically universal. This is the case of environmental law, which can be seen as the entirety of rules and judgements, but also customs that relate to nature. Substantive environmental law is most definitely found in codes of behaviour and all written normative texts. This is an important consideration, in particular for reasons of the effectiveness and usefulness of laws protecting the environment, but it is also interesting to discover its roots. We find these in the art of conceptualising and reflecting on law. This 
conceptualisation is linked to establishing environmental law as fundamental, understood as a process that develops the basis of the whole subject. The concepts become the components of advanced legal thinking that then becomes systematised. They are also infused with values, and show what is at the very heart of these laws.

\section{AUTEUR}

\section{PIERRE-YVES CHICOT}

Maître de conférences de droit public habilité à diriger des recherches, Centre de recherche en économie et en droit du développement insulaire (CREDDI), université des Antilles 